\title{
Effects of aging on the mechanical and dielectric properties of transformer grade Kraft paper
}

\author{
Ian L. Hosier, \\ Tony Davies High Voltage \\ Laboratory, \\ University of Southampton, \\ Southampton, SO17 1BJ, UK \\ ilh@soton.ac.uk
}

\author{
Paul L. Lewin, \\ Tony Davies High Voltage \\ Laboratory, \\ University of Southampton, \\ Southampton, SO17 1BJ, UK \\ pll@ecs.soton.ac.uk
}

\author{
James Pilgrim, \\ Tony Davies High Voltage \\ Laboratory, \\ University of Southampton, \\ Southampton, SO17 1BJ, UK \\ jp2@ecs.soton.ac.uk
}

\author{
Gordon Wilson, \\ National Grid, \\ Warwick Technology Park, \\ Gallows Hill, \\ Warwick, CV34 6DA, UK \\ gordon.wilson@nationalgrid.com
}

\begin{abstract}
In order to understand the aging process of paper used in high voltage transformers, transformer grade Kraft paper samples were aged in a fan oven in order to access the full range of DP values from 1100 (new) to 200 (end of life). The absence of significant oxidation was verified by infrared spectroscopy and the mechanical and dielectric properties were assessed as a function of DP. Whilst the dielectric properties (in the absence of water) were unaffected by aging, the tensile strength was reduced. This confirms studies in the literature which show that most transformer breakdowns occur through mechanical failure of the paper and crucially, provides a mechanism of providing paper samples of known DP for subsequent exposure to oil flow.
\end{abstract}

\section{Keywords—Transformer insulation, aging, DP}

\section{INTRODUCTION}

In a typical high voltage distribution transformer a series of copper coils are wound onto a magnetic core. To electrically isolate the individual phases from each other, pressboard is used, whilst to isolate the individual turns, they are wrapped with multiple layers of paper tape. Typically such windings are then formed into a series of stacked disks with hollow channels and the whole assembly is then dried and placed into a tank of mineral oil. During normal operation oil is permitted to flow, either by natural or forced convection, through the channels and heat is extracted from the windings which is then safely dissipated via a radiator into the environment [1]. Whilst the oil provides a vital cooling function, it is the aging of the paper that ultimately determines plant lifetime $[2,3]$. Crucially evidence obtained over a number of years by National Grid indicates that most transformer breakdowns occur due to mechanical failure of the paper [2]. Given the difficulty of accessing the paper in a live asset for direct testing [1], many indirect techniques such as dissolved gas analysis, furan analysis, dielectric spectroscopy, recovery voltage, acidity and water content are routinely employed to infer the condition of plant [3].

The degree of polymerisation (DP) is a useful indicator of the condition of insulation paper and typically new Kraft has a DP of $\sim 1000$ whilst a value of $\sim 200$ is usually taken to indicate end of life $[4,5]$. The dielectric properties of the paper are unchanged by extreme aging $[2,5]$ and are dominated largely by the effects of absorbed moisture [5]. However it is widely reported [2, 4, 6 - 9] that paper becomes mechanically embrittled after aging. Therefore it is not difficult to envisage a scenario whereby a high rate of oil flow, for example in switching from ONAN to OFAF operation during periods of high load or alternatively, through routine reclamation activities [10] could irreversibly damage the insulation system, compromising plant health. Alternatively the paper may not be able to withstand the normal electro-mechanical forces inherent to plant operation.

The current project seeks to understand the relationships between aging and mechanical failure of the paper when exposed to oil flows mimicking those found in plant. The first part of this investigation, reported here, concerns the preparation and characterisation of samples of known DP for future oil flow testing. Samples were aged in fan ovens to provide samples covering the full range of DP values and their mechanical and dielectric properties were then characterised.

\section{EXPERIMENTAL}

\section{A. Paper aging}

For subsequent flow testing it was required to have aged paper samples of known DP and water content for insertion into a mineral oil of known condition. Consequentially, transformer grade Kraft paper $(0.1 \mathrm{~mm}$ thickness $)$ was cut into $10 \times 10 \mathrm{~cm}$ pieces and hung on hooks in a fan oven maintained at $150{ }^{\circ} \mathrm{C}$. This temperature was chosen to ensure that the complete range of DP values was accessible within a reasonable timeframe whilst avoiding pyrolysis $[4,7,9]$. The use of a fan oven, rather than aging samples under oil, has several advantages such as; removal of aging by-products [3], keeping the paper in a dry state during aging [9], ease of subsequent sample analysis and eliminating any aging of the mineral oil.

\section{B. Characterisation}

After aging, all samples were left under ambient conditions $\left(25{ }^{\circ} \mathrm{C}, \mathrm{RH}=45 \pm 10 \%\right)$ for at least $24 \mathrm{~h}$ prior to testing. The moisture content was then inferred by drying to constant mass. Measurements of DP were performed according to ASTM D4243 [4, 7]; $15 \mathrm{mg}$ samples were dissolved in $20 \mathrm{ml}$ of $0.5 \mathrm{M}$ Bis(ethylenediamine) copper(II) hydroxide solution (Sigma Aldrich) through constant stirring in closed jars for $4 \mathrm{~h}$. The viscosity of the resulting solutions was then measured five times using a glass capillary viscometer placed into a water bath maintained at $20.0 \pm 0.5^{\circ} \mathrm{C}$ and the DP was then calculated. To check for oxidation, infrared spectroscopy was performed using a Thermoscientific Nicolet iS5 spectrometer fitted with an iD7 
ATR accessory. An average of 16 scans were taken and after taking a background, samples were scanned in quick succession to minimise any effects due to changes in ambient conditions. Mechanical testing was performed on cut strips nominally $70 \mathrm{x}$ $15 \mathrm{~mm}$ in size under ambient conditions. Since paper is mechanically anisotropic [6], strips were cut longitudinally (L, along the fibre direction) and in the transverse direction ( $\mathrm{T}$, perpendicular to the fibres) for testing. The machine used was a Tinius Olsen H25KS Tensometer, the gauge length was $50 \mathrm{~mm}$, the crosshead speed was $10 \mathrm{~mm} / \mathrm{min}$ and all samples were pulled to failure. Dielectric spectroscopy was performed on $50 \times 50$ $\mathrm{mm}$ samples placed in a test cell composed of opposing $50 \mathrm{~mm}$ diameter gold plated electrodes. Tests were performed under ambient and dry conditions, the latter being achieved by first drying the paper to constant mass then performing all tests within a desiccator $(\mathrm{RH}<10 \%)$. A Schlumberger SI 1260A impedance gain-phase analyser was connected to a Solartron 1296 dielectric interface and an $\mathrm{AC}$ voltage of $3 \mathrm{~V}_{\mathrm{rms}}$ and integration period of $10 \mathrm{~s}$ was used for all measurements.

\section{RESULTS}

\section{A. Water absorption kinetics}

The drying behaviour of new paper samples at various temperatures is shown in Fig. 1a, each plot is the average of measurements made on 5 independent samples. As indicated by the exponential fitted lines, the drying time (to constant mass)
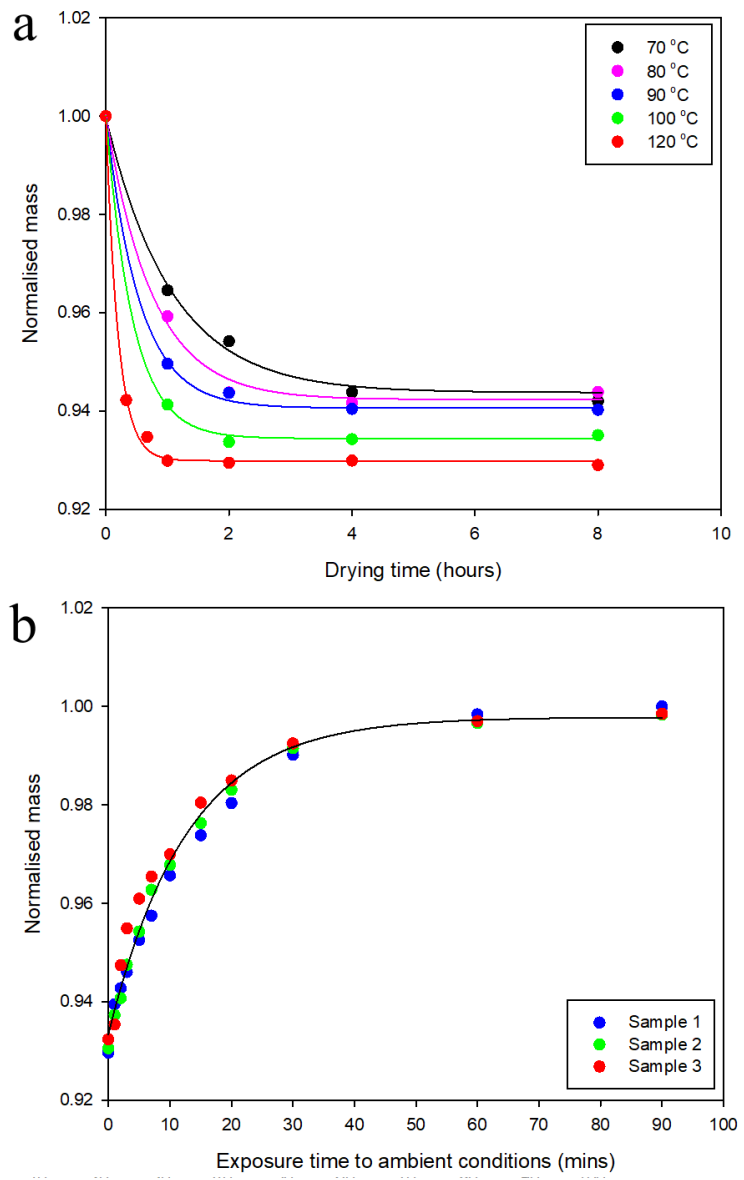

Fig. 1. Water; (a) desorption on drying, (b) absorption following exposure of dried paper to ambient conditions. varies between $6 \mathrm{~h}$ and $1 \mathrm{~h}$ as the temperature is increased and the loss in mass corresponds to a water content of between 5.8 and $7.1 \mathrm{wt} . \%$. The latter value is consistent with the literature [5] which suggests that $120{ }^{\circ} \mathrm{C}$ is the most suitable drying temperature. Next, three independent samples which had been fully dried $\left(120^{\circ} \mathrm{C}\right.$ for $2 \mathrm{~h}$ ) were left under ambient conditions and their subsequent water uptake was followed (Fig. 1b). As indicated by the fitted line, the samples quickly regain their water content of $\sim 7$ wt. $\%$ within $1 \mathrm{~h}$; this indicates that some care needs to be taken when performing dielectric spectroscopy measurements on dried paper samples to ensure that water is not reabsorbed during testing.

\section{B. Effects of aging on DP}

Fig. 2a shows measurements of DP as a function of aging time for 3 independent sets of samples and as expected, the full range of DP values could be accessed within $500 \mathrm{~h}[4,7,9]$. Elsewhere [2] an equation for the dependence of DP $\left(D P_{t}\right)$ on aging time $(t)$ was derived from first principles;

$$
\frac{1}{D P_{t}}-\frac{1}{D P_{o}}=\frac{k_{1}}{k_{2}}\left(1-e^{-k_{2} t}\right)
$$

where $D P_{o}$ is the DP at time $t=0$ and $k_{1}$ and $k_{2}$ are constants. The DP data were taken and used to calculate the parameter on the left hand side of the equation (Fig. 2b). An appropriate 2 parameter exponential fit was then applied to the entire dataset as indicated by the solid line. The good correlation $\left(\mathrm{R}^{2}>0.994\right)$
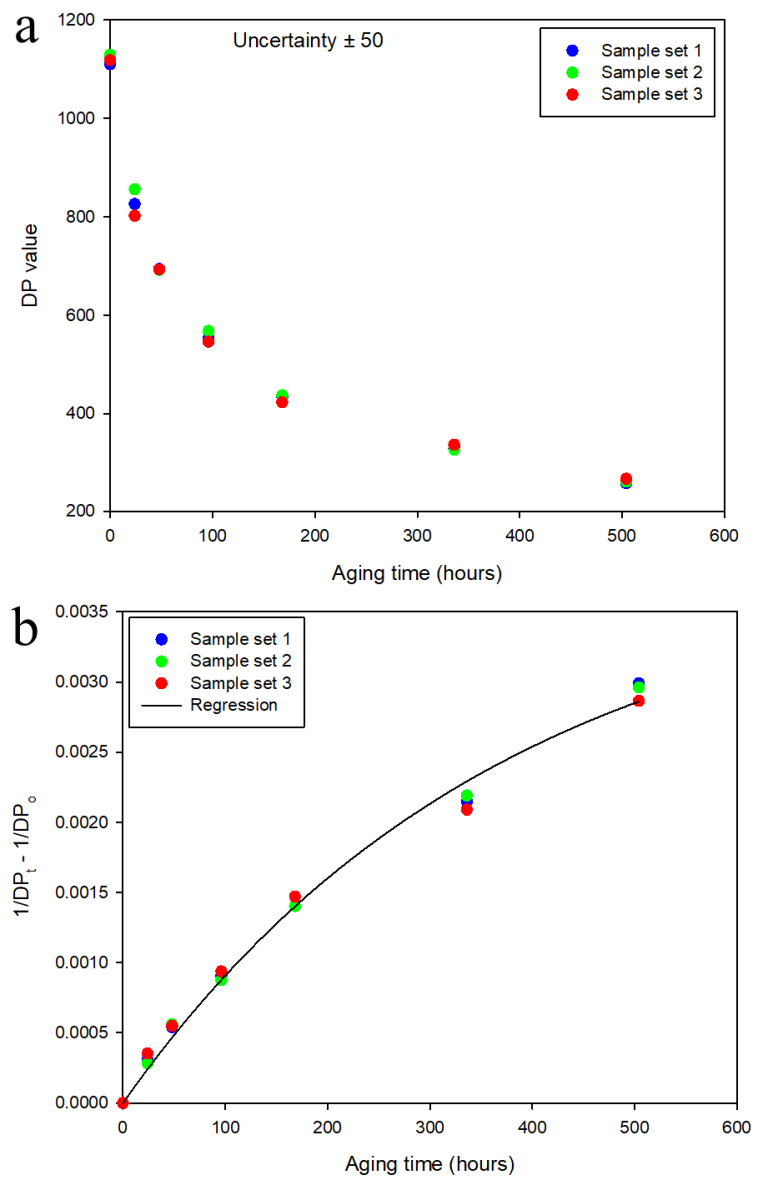

Fig. 2. DP measurements; (a) as collected data, (b) fit to theory. 
indicates that the aging process follows established theory confirming the validity of the aging protocol used here.

\section{Infrared spectroscopy}

Typical infrared absorption spectra are shown in Fig. 3 and the positions of the major absorptions corresponding to the various chemical bonds present in cellulose (inset) are indicated [11]. The peak at $\sim 1000 \mathrm{~cm}^{-1}$ corresponding to glycosidic bonds (C-O-C) is weakened reflecting the observed reduction in DP. The only other obvious change due to aging is the presence of a minor feature at $\sim 1720 \mathrm{~cm}^{-1}$ (arrowed) which most likely arises from the formation of carboxylic acid groups during aging [3, 11]. Crucially the spectra show that, even at this high aging temperature, oxidation is minimal.

\section{Effect of aging on mechanical properties}

Typical results from tensile testing in the longitudinal and transverse directions is shown in Figs. $4 \mathrm{a}$ and $4 \mathrm{~b}$. The tensile strength is higher in the fiber direction (Fig. 4a) as expected [6] and both plots confirm an embrittlement of the paper due to aging $[2,4,6-9]$. To quantify this change, the strain at break was taken and plotted against the aging time (Fig. 4c). The behavior is consistent with the literature [8] and indicates for DP $<300$ (aging time $>400 \mathrm{~h}$ ) the break strain is $<1 \%$ and at this point the paper would be considered to be at the end of life [4]. Thus it is possible here to prepare samples for flow testing covering the full range of available mechanical properties.

\section{E. Effects of aging on dielectric properties}

Fig. 5a and 5b show the results from measurements of relative permittivity and dielectric loss $(\tan \delta)$ after different aging times. In ambient conditioned samples (solid symbols), which will contain appreciable water, the permittivity shows an uplift at low frequencies (Fig. 5a) and the dielectric loss (Fig. 5 b) shows a mid-frequency relaxation $(\sim 3 \mathrm{~Hz})$ along with a low frequency uplift. Such features are consistent with results presented elsewhere for paper samples containing comparable amounts of water [5]. Interestingly, under dry conditions (open symbols) such features entirely disappear and elsewhere [5] results for $0.2 \mathrm{wt}$ \% water approach this condition, thus the observed effects are solely due to absorbed water. Crucially, when the water is taken away, there is no indication here, within

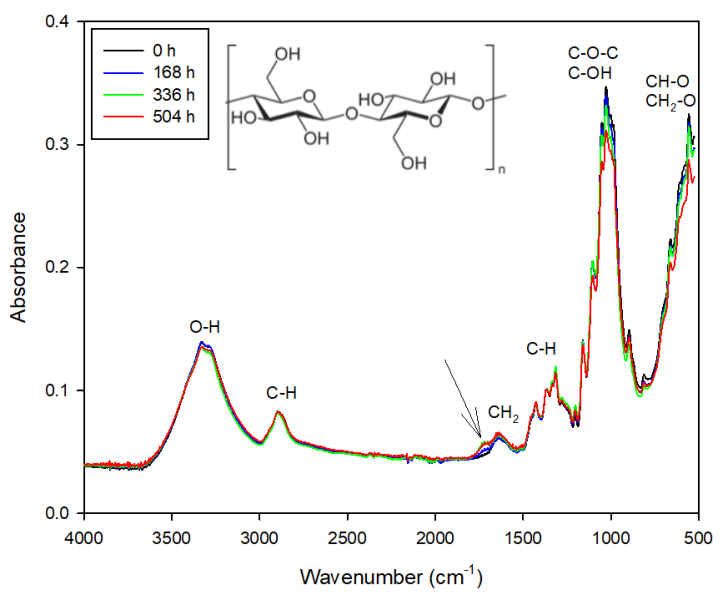

Fig. 3. Typical set of infrared spectra from new and aged paper samples.
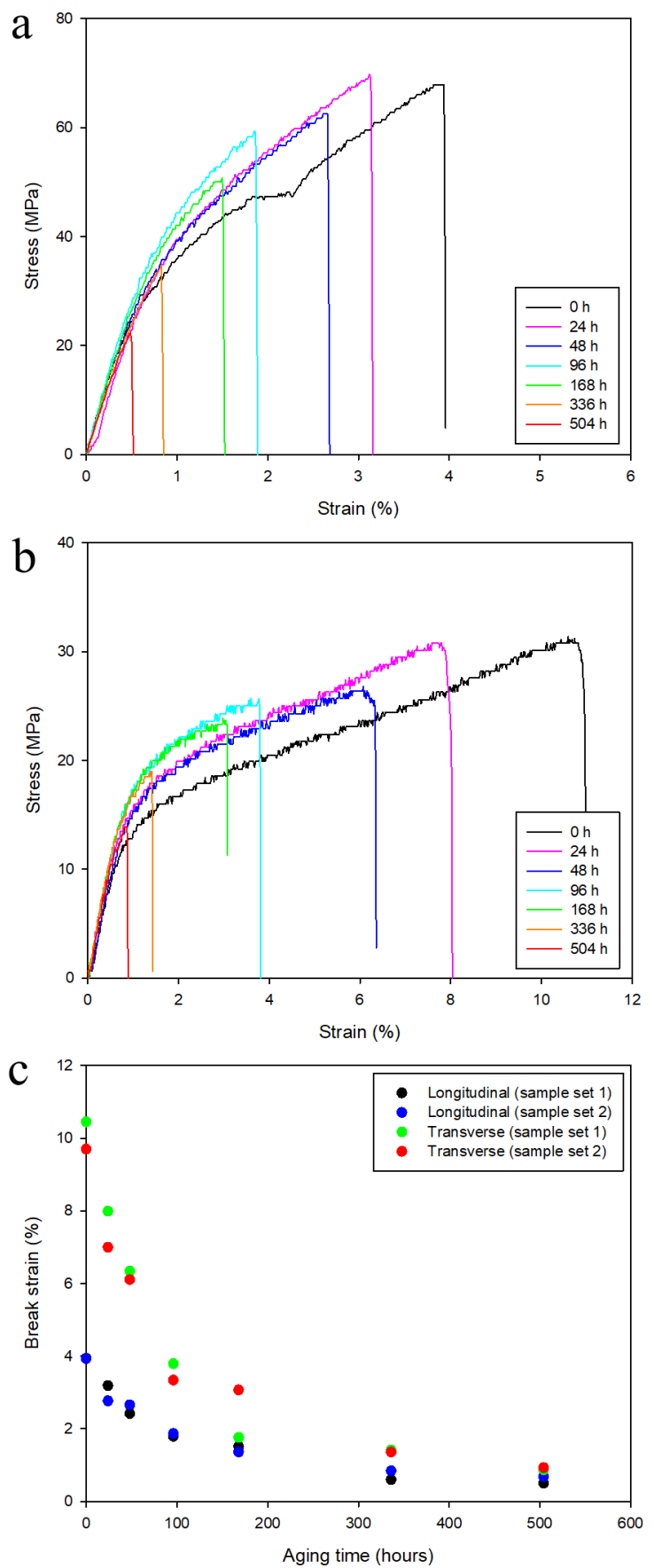

Fig. 4. Results from tensile testing; (a) longitudinal direction, (b) transverse direction, (c) summary of break strain against aging time.

the sensitivity limits of our equipment, that aging is having any significant effect on the dielectric properties as expected $[2,5]$.

Nevertheless water is a useful probe and under ambient conditions, it is clear that there is an apparent improvement in the dielectric properties after aging. It has been shown elsewhere that aging reduces the propensity of paper to absorb water [9] and this effect would be manifested here (under conditions of constant humidity) as an apparent improvement. To confirm this, the water content was directly measured (Fig. 

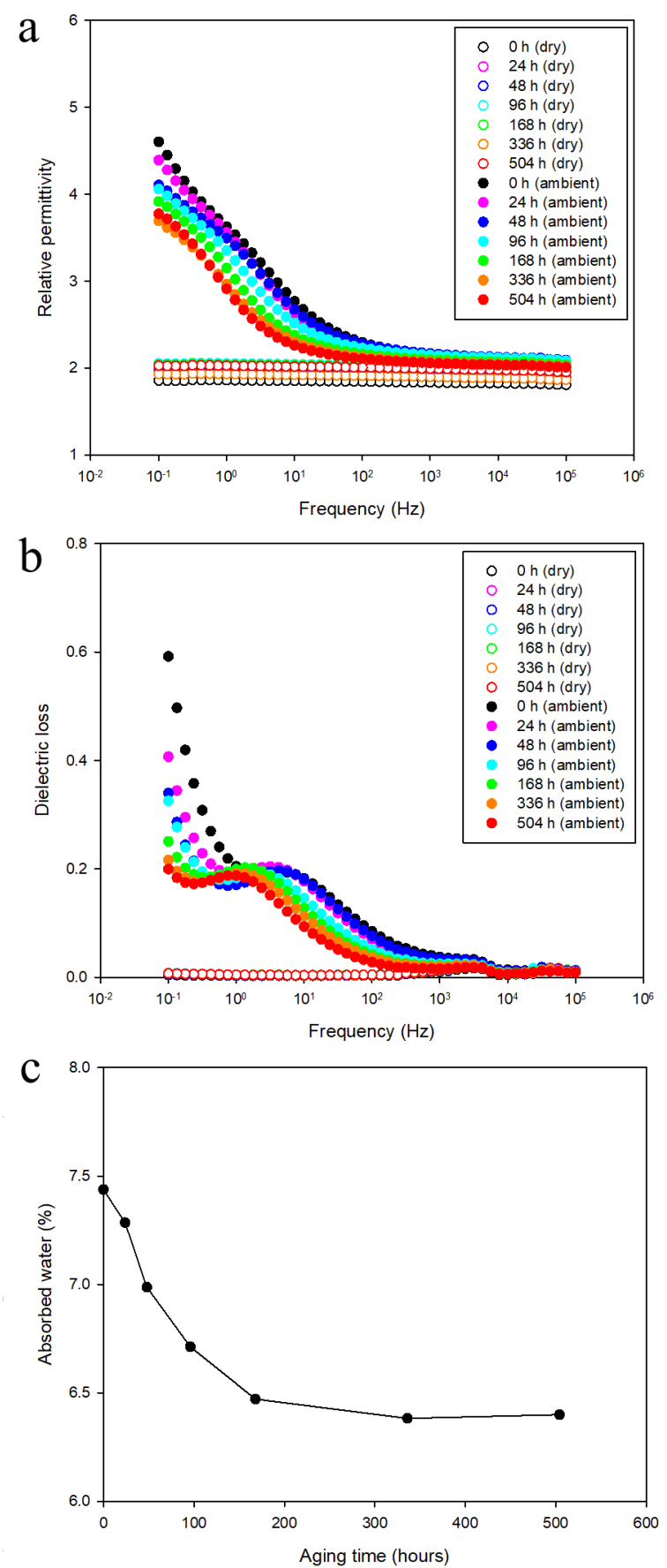

Fig. 5. Results from dielectric spectroscopy measurements; (a) relative permittivity, (b) dielectric loss, (c) water absorption in ambient conditioned samples (uncertainty $\pm 0.1 \%$ ).

5c) and the data confirm a drop in the absorbed water content of up to $1 \mathrm{wt}$. \% following aging which is more than sufficient to cause the observed variations [5].

\section{CONCLUSIONS}

Samples of transformer grade Kraft paper were aged in fan ovens at $150{ }^{\circ} \mathrm{C}$. The full range of DP values from new to end of life could be accessed within a convenient $(500 \mathrm{~h})$ timescale and the observed change in DP with aging time fits established theory. Infrared spectroscopy confirms that oxidation under these conditions is minimal which supports the validity of the current approach.

Measurements of tensile strength confirm that the paper is embrittled by aging and more crucially, that the full range of available mechanical properties is accessible through the current aging protocol. Dielectric spectroscopy results confirm the critical role of absorbed water and in dry paper, demonstrate that aging, even under these extreme conditions, does not significantly change the dielectric properties of the paper.

The current aging regime therefore provides a method to provide samples of known DP covering the full range of mechanical properties which is crucial for future flow testing. In parallel with such testing it is planned to carry out aging studies at lower temperatures which are more representative of those found in a transformer. Such work will be reported in due course.

\section{ACKNOWLEDGMENT}

We would like to thank National Grid for providing financial support and for giving us permission to publish these results.

\section{REFERENCES}

[1] P. J. Baird, H. Herman, G. C. Stevens, P. N. Jarman, "Non-Destructive Measurement of the Degradation of Transformer Insulating Paper", IEEE Trans. Diel. Electr. Insul., vol. 13, pp. 309-318,2006.

[2] A. M. Emsley, R. J. Heywood, M. Ali, and X. Xiao, "Degradation of cellulosic insulation in power transformers. Part 4: Effects of ageing on the tensile strength of paper," IEEE Proceedings-Science Measurement and Technology, vol. 147, pp. 285-290, 2000.

[3] M. Wang, A. J. Vandermaar, and K. D. Srivastava, "Review of condition assessment of power transformers in service, " IEEE Electr. Insul. Mag., vol. 18, pp. 12-25, 2002.

[4] L. E. Lundgaard, W. Hansen, D. Linhjell, T. J. Painter, "Aging of OilImpregnated Paper in Power Transformers", IEEE Trans. Pow. Del., vol. 19, pp. 230-239, 2004.

[5] D. Linhjell, L. Lundgaard, U. Gäfvert, "Dielectric Response of Mineral Oil Impregnated Cellulose and the Impact of Aging", IEEE Trans. Diel. Electr. Insul., vol. 14, pp. 156-169,2007.

[6] D. J. T. Hill, T. T. Le, M. Darvenizab, T. Saha, "A study of degradation of cellulosic insulation materials in a power transformer. Part 2:tensile strength of cellulose insulation paper", Polym. Degr. Stab., vol. 49, pp. 429-435, 1995.

[7] I. A. Carrascal, C. Fernández-Diego, J. A. Casado, S. Diego, I. Fernández, A. Ortiz, "Quantification of Kraft paper ageing in mineral oil impregnated insulation systems through mechanical characterization", Cellulose, vol. 25, pp. 3583-3594, 2018.

[8] C. Fernaández-Diego, A. Ortiz, I. A. Carrascal . I. Fernández, C. J. Renedo, F. Delgado, S. Diego, "Damage assessment of transformer Kraft paper insulation aged in mineral and vegetable oils", Cellulose, vol. 26, pp. 2653-2672, 2019.

[9] K. L. Kato, R. E. Cameron, "A review of the relationship between thermally-accelerated ageing of paper and hornification", Cellulose, vol. 6, pp. 23-40, 1999.

[10] R. P. Venkatasubramanian, "Experimental Assessment of Oil Regeneration Technique for Transformer Life Extension", PhD Thesis, University of Manchester, 2015, p. 67.

[11] R. Saldivar-Guerrero, E. N. Cabrera Álvarez, U. Leon-Silva, F. A. LopezGonzalez, F. Delgado Arroyo, H. Lara-Covarrubias, R. MontesFernandez, "Quantitative Analysis of Ageing Condition of Insulating Paper Using Infrared Spectroscopy", Adv. Mater. Sci. Eng., Art. No. 6371540 (5 pp.), 2016 